\title{
High expression of miR-25 predicts favorable chemotherapy outcome in patients with acute myeloid leukemia
}

Mingshan Niu ${ }^{1,2+}$, Yuan Feng ${ }^{1 \dagger}$, Ninghan Zhang ${ }^{1 \dagger}$, Tingting Shao ${ }^{1}$, Huihui Zhang ${ }^{1}$, Rong Wang ${ }^{1}$, Yao Yao ${ }^{1,2}$,

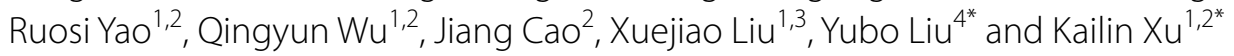

\begin{abstract}
Background: Acute myeloid leukemia (AML) pertains to a hematologic malignancy with heterogeneous therapeutic responses. Improvements in risk stratification in AML patients are warranted. MicroRNAs have been associated with the pathogenesis of AML.

Methods: To examine the prognostic value of miR-25, 162 cases with de novo AML were classified into two groups according to different treatment regimens.

Results: In the chemotherapy group, cases with upregulated miR-25 expression showed relatively longer overall survival $(O S ; P=0.0086)$ and event-free survival $(E F S ; P=0.019)$. Multivariable analyses revealed that miR-25 upregulation is an independent predictor for extended OS ( $H R=0.556, P=0.015)$ and $E F S(H R=0.598, P=0.03)$. In addition, allogeneic hematopoietic stem cell transplantation (allo-HSCT) circumvented the poor prognosis that was related to miR-25 downregulation with chemotherapy. The expression level pattern of miR-25 coincided with AML differentiation and proliferation, which included HOXA and HOXB cluster members, as well as the HOX cofactor MEIS1. The MYH9 gene was identified as a direct target of miR-25.
\end{abstract}

Conclusions: The miR-25 levels are correlated with prognosis in AML independently of other powerful molecular markers. The expression of miR-25 may contribute to the selection of the optimal treatment regimen between chemotherapy and allo-HCST for AML patients.

Keywords: Acute myeloid leukemia, miR-25, Clinical outcome, Chemotherapy, Allo-HSCT

\section{Background}

Acute myeloid leukemia (AML) is a group of clonal malignant diseases that derive from the hematopoietic stem cells. AML is characterized by a large group of germinal cells, which leads to a loss of normal hematopoietic function [1]. The clinical prognosis of patients with AML is various. The differences in outcomes among AML patients depend on multiple intrinsic factors $[2,3]$. With

\footnotetext{
*Correspondence: liuyubo@dlut.edu.cn; lihmdl@163.com

${ }^{\dagger}$ Mingshan Niu, Yuan Feng and Ninghan Zhang contributed equally to this work

${ }^{1}$ Blood Diseases Institute, Affiliated Hospital of Xuzhou Medical University, Xuzhou Medical University, Xuzhou, Jiangsu, China

${ }^{4}$ School of Life Science \& Medicine, Dalian University of Technology, Panjin, China

Full list of author information is available at the end of the article
}

the development of methodologies of massive sequencing, it has been demonstrated that somatic mutations in NPM1, FLT3, CEBPA, IDH1 and IDH2 are connected to prognosis in AML [4]. To be specific, patients with mutated FLT3 have a dismal outcome, while mutations in NPM1 and CEBPA are related with favorable prognosis. The advent of chemotherapy and allogeneic hematopoietic stem cell transplantation (allo-HSCT) has significantly improved AML treatment outcomes [5]. Relapse and refractory of leukemia remain the most disturbing problems in AML patients [6]. Thus, it is urge to explore more reliable and effective prognostic biomarkers to enhance the capacity of prediction and thus improve the outcome of AML by choosing optimal therapeutic approach.

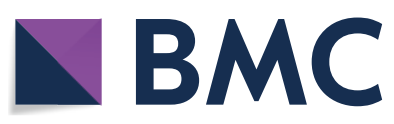

(c) The Author(s) 2019. This article is distributed under the terms of the Creative Commons Attribution 4.0 International License (http://creativecommons.org/licenses/by/4.0/), which permits unrestricted use, distribution, and reproduction in any medium, provided you give appropriate credit to the original author(s) and the source, provide a link to the Creative Commons license, and indicate if changes were made. The Creative Commons Public Domain Dedication waiver (http://creativecommons.org/ publicdomain/zero/1.0/) applies to the data made available in this article, unless otherwise stated. 
MicroRNAs are short non-coding RNAs, which are implicated in a diverse group of critical cellular mechanisms, such as apoptosis, differentiation, cell cycle progression, and immune responses [7]. Recently, more and more attentions have been focused on the prognostic role of microRNAs in AML. A recent study has shown that the upregulation of miR-181a facilitates better survival of AML patients who are cytogenetically normal [8]. However, AML patients who are cytogenetically normal and upregulated miR-212 and miR-3151 have shorter overall and disease-free survival $[9,10]$. However, most microRNA analyses did not differentiate the AML patients treated with chemotherapy and allo-HSCT. Thus, microRNAs may have varied prognostic roles in chemotherapy and allo-HSCT treatment group, respectively.

MiR-25, a member of miR-106b-25 cluster, is located on human chromosome 7q22.1 [11]. Previous studies revealed that miR-25 was involved in many kinds of cancers [12]. It has been identified that miR-25 is a potential biomarker for pediatric AML based on Pipeline of Outlier MicroRNA Analysis (POMA) model [13]. More important, Garzon et al. [14] reported that miR-25 is significantly down-regulated in 122 newly diagnosed AML samples compared with CD $34^{+}$normal cells. However, clinical and prognostic role of miR-25 in AML are still unclear. A total of 162 recently diagnosed de novo AML patients were enrolled in this evaluation. The cases were placed into two groups based on the treatment that they received. The present study suggested that miR-25 is a solitary AML prognostic biomarker. Furthermore, our study revealed that allo-HSCT would be more beneficial to patients showing downregulated miR-25.

\section{Patients and methods Patients}

Approximately 162 patients with a diagnosis of de novo AML were included in this study. The data sets used in this investigation were acquired from The Cancer Genome Atlas (TCGA). A single-institution tissue banking strategy endorsed by the human studies committee of Washington University was used in this study. All of the patients provided their written informed consent. AML diagnosis and classification were made according to the French-American-British (FAB) and the World Health Organization (WHO) criteria. The cases were placed into two groups according to the clinical treatment received. Ninety patients accepted chemotherapy, and the rest accepted allo-HSCT.

\section{Gene-expression profiling}

The samples from 155 patients both had been obtained mRNA and microRNA expression data. These data were applied to identify the mRNA-expression signature associated with miR-25 expression. The sequencing read count for each miRNA was normalized to Reads per million reads (RPM). The mRNA expression values were logged (base 2) prior to analysis [15]. Spearman correlation was used to correlate the mRNA-expression profile with miR-25 expression. Hierarchical clustering analysis was used to reorder the gene rows. To screen for target genes of miR-25, Targetscan, miRNApath and miRDB website tools were implemented. Gene Ontology enrichment assessment of genes in miR-340 related signature was performed with the Database for Annotation, Visualization, and Integrated Discovery (DAVID).

\section{Statistical analysis}

The clinical endpoints of this investigation on treatment outcomes included overall survival (OS) and event-free survival (EFS). OS pertains to the time interval from diagnosis to death or last follow-up of the patient. EFS is described as the time interval from diagnosis to disease progression, relapse, or death attributed to any cause. The patients were assigned to the high or low expression groups based on the median miR-25 expression. Descriptive statistics (median and/or range) were used to summarize patients' clinical and molecular characteristics. To elucidate the role of miR-25 expression in AML clinical and molecular features, the Pearson Chi-square and Fisher's exact tests were used to screen for significant differences between two categorical variables. In addition, the Mann-Whitney's $U$ test was used for continuous variables. For univariable and multivariable analysis, a Cox proportional hazards model was employed to determine the effect of various risk factors on patient OS and EFS. The limited backward elimination procedure was applied to assess hazard ratios (HRs) and $P$ values. Kaplan-Meier analysis was performed to determine the impact of miR25 expression on OS and EFS. Statistical analysis was conducted with SPSS and GraphPad Prism. Differences among variables were determined to be statistically significant when the $P$ value was $<0.05$.

\section{Results \\ Correlation analysis of miR-25 expression and clinical characteristics}

To establish the correlation among miR-25 expression and various clinical profiles, we assigned the patients who underwent chemotherapy and allo-HSCT to one of two groups according to median miR-25 expression levels, respectively. The associations of the clinical features with miR-25 expression levels are summarized in Table 1. In the chemotherapy group, subjects who exhibited upregulated miR-25 had a higher percentage of RUNX1RUNX1T1 compared to those with downregulated expression $(P=0.026)$. In addition, high miR-25 expresser 
Table 1 Comparison of clinical and molecular characteristics with miR-25 expression in patients with AML

\begin{tabular}{|c|c|c|c|c|c|c|}
\hline \multirow[t]{2}{*}{ Characteristic } & \multicolumn{3}{|c|}{ Chemotherapy group } & \multicolumn{3}{|c|}{ Allo-HSCT group } \\
\hline & $\begin{array}{l}\text { High miR-25 } \\
(n=45)\end{array}$ & $\begin{array}{l}\text { Low miR-25 } \\
(n=45)\end{array}$ & $P$ & $\begin{array}{l}\text { High miR-25 } \\
(n=36)\end{array}$ & $\begin{array}{l}\text { Low miR-25 } \\
(n=36)\end{array}$ & $P$ \\
\hline Age/years, median & $61.4(22-82)$ & $64.4(31-88)$ & 0.49 & $47.3(22-72)$ & $49.4(18-69)$ & 0.535 \\
\hline Age group/n (\%) (years) & & & 0.495 & & & 0.793 \\
\hline$<60$ & $16(35.6)$ & $12(26.7)$ & & $27(75)$ & $25(69.4)$ & \\
\hline$\geq 60$ & $29(64.4)$ & $33(73.3)$ & & $9(25)$ & $11(30.6)$ & \\
\hline Gender/n (\%) & & & 0.289 & & & 0.634 \\
\hline Male & $22(48.9)$ & $28(62.2)$ & & $22(61.1)$ & $19(52.8)$ & \\
\hline Female & $23(51.1)$ & $17(37.8)$ & & $14(38.9)$ & $17(47.2)$ & \\
\hline WBC/× 109/L, median & $32.4(0.7-297.4)$ & $51.8(1.5-298.4)$ & 0.059 & $36.0(0.6-223.8)$ & $39.8(1.2-118.8)$ & 0.248 \\
\hline BM blast/\%, median & $69(32-99)$ & $67.2(30-92)$ & 0.955 & $66.2(34-99)$ & $70.2(30-100)$ & 0.277 \\
\hline PB blast/\%, median & $39.5(0-98)$ & $35.1(0-97)$ & 0.320 & $46.1(0-96)$ & $48.9(0-94)$ & 0.752 \\
\hline \multicolumn{7}{|l|}{ FAB subtypes/n (\%) } \\
\hline MO & $4(8.9)$ & $4(8.9)$ & 1.000 & $6(16.7)$ & $3(8.3)$ & 0.478 \\
\hline M1 & $13(28.9)$ & $7(15.6)$ & 0.204 & $7(19.4)$ & $16(44.4)$ & 0.042 \\
\hline M2 & $12(26.7)$ & $9(20)$ & 0.619 & $12(33.3)$ & $7(19.4)$ & 0.285 \\
\hline M4 & $11(24.4)$ & $13(28.9)$ & 0.812 & $8(22.2)$ & $6(16.7)$ & 0.767 \\
\hline M5 & $4(8.9)$ & $9(20)$ & 0.230 & $2(5.6)$ & $2(5.6)$ & 1.000 \\
\hline M6 & $1(2.2)$ & $0(0.0)$ & 1.000 & $0(0.0)$ & $1(2.8)$ & 1.000 \\
\hline M7 & $0(0.0)$ & $2(4.4)$ & 0.494 & $1(2.8)$ & $0(0.0)$ & 1.000 \\
\hline No date & $0(0.0)$ & $1(2.2)$ & 1.000 & $0(0.0)$ & $1(2.8)$ & 1.000 \\
\hline \multicolumn{7}{|l|}{ Karyotype/n (\%) } \\
\hline Normal & $18(40)$ & $26(57.8)$ & 0.140 & $15(41.7)$ & $19(52.7)$ & 0.479 \\
\hline Complex & $5(11.1)$ & $7(15.6)$ & 0.758 & $6(16.6)$ & $6(16.6)$ & 1.000 \\
\hline Poor & $0(0.0)$ & $5(11.1)$ & 0.056 & $1(2.8)$ & $4(11.1)$ & 0.357 \\
\hline Intermediate & $8(17.8)$ & $2(4.4)$ & 0.090 & $6(16.7)$ & $3(8.4)$ & 0.478 \\
\hline$M L L$ & $1(2.2)$ & $2(4.4)$ & 1.000 & $3(8.3)$ & $0(0.0)$ & 0.239 \\
\hline CBF $\beta-M Y H 11$ & $6(13.3)$ & $1(2.2)$ & 0.110 & $4(11.1)$ & $1(2.8)$ & 0.357 \\
\hline BCR-ABL1 & $0(0.0)$ & $1(2.2)$ & 1.000 & $0(0.0)$ & $2(5.6)$ & 0.493 \\
\hline RUNX1-RUNX1T1 & $6(13.3)$ & $0(0.0)$ & 0.026 & $1(2.8)$ & $0(0.0)$ & 1.000 \\
\hline N.D. & $1(2.2)$ & $1(2.2)$ & 1.000 & $0(0.0)$ & $1(2.8)$ & 1.000 \\
\hline \multicolumn{7}{|l|}{ Risk(cyto)/n (\%) } \\
\hline Good & $12(26.7)$ & $1(2.2)$ & 0.002 & $5(13.9)$ & $1(2.8)$ & 0.199 \\
\hline Intermediate & $26(57.8)$ & $29(64.4)$ & 0.665 & $21(58.3)$ & $20(55.5)$ & 1.000 \\
\hline Poor & $6(13.3)$ & $14(31.1)$ & 0.074 & $10(27.8)$ & $14(38.9)$ & 0.454 \\
\hline Other & $1(2.2)$ & $1(2.2)$ & 1.000 & $0(0.0)$ & $1(2.8)$ & 1.000 \\
\hline FLT3-ITD/n (\%) & & & 0.784 & & & 0.045 \\
\hline Presence & $9(20.0)$ & $7(15.6)$ & & $4(11.1)$ & $12(33.3)$ & \\
\hline Absence & $36(80.0)$ & $38(84.4)$ & & $32(88.9)$ & $24(66.7)$ & \\
\hline NPM1/n (\%) & & & 0.175 & & & 0.064 \\
\hline Presence & $11(24.4)$ & $18(40)$ & & $6(16.7)$ & 14 (38.9) & \\
\hline Absence & 34 (75.6) & $27(60)$ & & $36(83.3)$ & $22(61.1)$ & \\
\hline DNMT3A/n (\%) & & & 0.157 & & & 1.000 \\
\hline Presence & $9(20)$ & $16(35.6)$ & & $9(25)$ & $9(25)$ & \\
\hline Absence & $36(80)$ & $29(64.6)$ & & $27(75)$ & $27(75)$ & \\
\hline RUNX1/n (\%) & & & 0.714 & & & 0.710 \\
\hline Presence & $5(11.1)$ & $3(6.7)$ & & $5(13.9)$ & $3(8.3)$ & \\
\hline Absence & 40 (89.9) & $42(93.3)$ & & $31(86.1)$ & $33(91.7)$ & \\
\hline MLL-PTD/n (\%) & & & 1.000 & & & \\
\hline
\end{tabular}


Table 1 (continued)

\begin{tabular}{|c|c|c|c|c|c|c|}
\hline \multirow[t]{2}{*}{ Characteristic } & \multicolumn{3}{|c|}{ Chemotherapy group } & \multicolumn{3}{|c|}{ Allo-HSCT group } \\
\hline & $\begin{array}{l}\text { High miR-25 } \\
(n=45)\end{array}$ & $\begin{array}{l}\text { Low miR-25 } \\
(n=45)\end{array}$ & $P$ & $\begin{array}{l}\text { High miR-25 } \\
(n=36)\end{array}$ & $\begin{array}{l}\text { Low miR-25 } \\
(n=36)\end{array}$ & $P$ \\
\hline Presence & $2(4.4)$ & $3(6.7)$ & & $2(5.6)$ & $2(5.6)$ & \\
\hline Absence & $43(95.6)$ & $42(93.3)$ & & $34(94.4)$ & $34(94.4)$ & \\
\hline TP53/n (\%) & & & 0.522 & & & 1.000 \\
\hline Mutation & $4(8.9)$ & $7(15.6)$ & & $2(5.6)$ & $2(5.6)$ & \\
\hline Wild type & $41(91.1)$ & $38(84.4)$ & & $34(94.4)$ & $34(94.4)$ & \\
\hline CEBPA/n (\%) & & & 1.000 & & & 0.055 \\
\hline Mutation & $1(2.2)$ & $2(4.4)$ & & $7(19.4)$ & $1(2.8)$ & \\
\hline Wild type & $44(97.8)$ & $43(95.6)$ & & $29(80.6)$ & $35(97.2)$ & \\
\hline IDH1/n (\%) & & & 1.000 & & & 0.514 \\
\hline Mutation & $3(6.7)$ & $4(8.9)$ & & $4(11.1)$ & $7(19.4)$ & \\
\hline Wild type & $42(93.3)$ & $41(91.1)$ & & $32(88.9)$ & $29(80.6)$ & \\
\hline IDH2/n (\%) & & & 1.000 & & & 0.260 \\
\hline Mutation & $5(11.1)$ & $4(8.9)$ & & $2(5.6)$ & $6(16.7)$ & \\
\hline Wild type & $40(88.9)$ & $41(91.1)$ & & $34(94.4)$ & $30(83.3)$ & \\
\hline
\end{tabular}

Mann-Whitney test was used for continuous variables. Chi square tests were used for categorical variables $W B C$ white blood cell, $B M$ bone marrow, $P B$ peripheral blood, FAB French-American-British classification

involved in more good risk cases of AML $(P=0.002)$. However, no significant differences were observed in gender, age, WBC count, BM blast, PB blast, FAB subtypes, FLT3-ITD, NPM1, DNMT3A, RUNX1, MLL-PTD, TP53, IDH1 and IDH 2 among the high and low miR-25 expression group. In the allo-HSCT group, study participants with upregulated miR-25 exhibited a lower frequency for FLT3-ITD mutations $(P=0.045)$ compared to those with downregulated miR-25. No significant differences in as far as gender, age, WBC count, BM blast, PB blast, and mutations in the NPM1, RUNX1, DNMT3A, MLL-PTD, IDH1, IDH2, and TP53 genes were observed among the upregulated and downregulated miR-25 groups.

\section{Prognostic value of miR-25 profiles in AML patients}

We performed Kaplan-Meier analysis and log-rank test to assess the prognostic value of miR-25 profiles in AML patients. The chemotherapy group showed that AML patients with upregulated miR-25 were connected to better EFS $(P=0.019)$ and OS $(P=0.0086)$ relative to those with downregulated miR-25 (Fig. 1a, b). However, AML patients who received allo-HSCT did not exhibit any connection among prognosis and miR-25 expression (Fig. 1c, d). These findings revealed that miR-25 may be utilized as a chemotherapy-specific prognostic marker for AML.

\section{High level of miR-25 is independently associated with favorable prognosis}

To determine whether miR-25 expression could be used as an independent predictor for AML patient survival, we conducted univariate and multivariate Cox analyses. For the chemotherapy group, univariate analysis revealed that the upregulation of miR-25 was connected with longer EFS (HR $=0.598,95 \%$ CI $0.376-0.951, P=0.030)$ and OS (HR $=0.556,95 \%$ CI $0.347-0.890, P=0.015)$. Furthermore, multivariate cox analysis indicated that miR-25 upregulation was independently connected with longer EFS $(\mathrm{HR}=0.561,95 \%$ CI $0.333-0.943, P=0.029)$ and $\mathrm{OS}(\mathrm{HR}=0.502,95 \% \mathrm{CI} 0.296-0.851, P=0.011)$ after adjustment of mutation status for the FLT3-ITD, NPM1, DNMT3A, RUNX1, IDH1, and IDH2 genes and WBC count (Table 2).

Univariate analysis of the allo-HSCT group suggested that AML cases harboring FLT3-ITD mutations had shorter EFS $(\mathrm{HR}=1.873,95 \%$ CI 1.020-3.437, $P=0.043)$ and $\mathrm{OS}(\mathrm{HR}=1.998,95 \%$ CI $1.053-3.788, P=0.034)$. Patients with mutations only in the RUNX1 gene exhibited shorter OS $(\mathrm{HR}=2.253,95 \%$ CI 1.046-4.849, $P=0.038)$. Multivariate analysis indicated that FLT3-ITD and RUNX1 remained independent outcome predictors after adjusting for all other prognostic factors (Table 3). However, allo-HSCT patients did not show any significant differences between upregulated and downregulated miR-25 expression.

\section{Allo-HSCT may circumvent poor patient outcomes that are related to downregulated $\mathrm{miR}-25$ expression}

To determine whether allo-HSCT therapy could circumvent the severe prognosis that was associated with downregulated miR-25, the whole cohort of 162 cases was split 

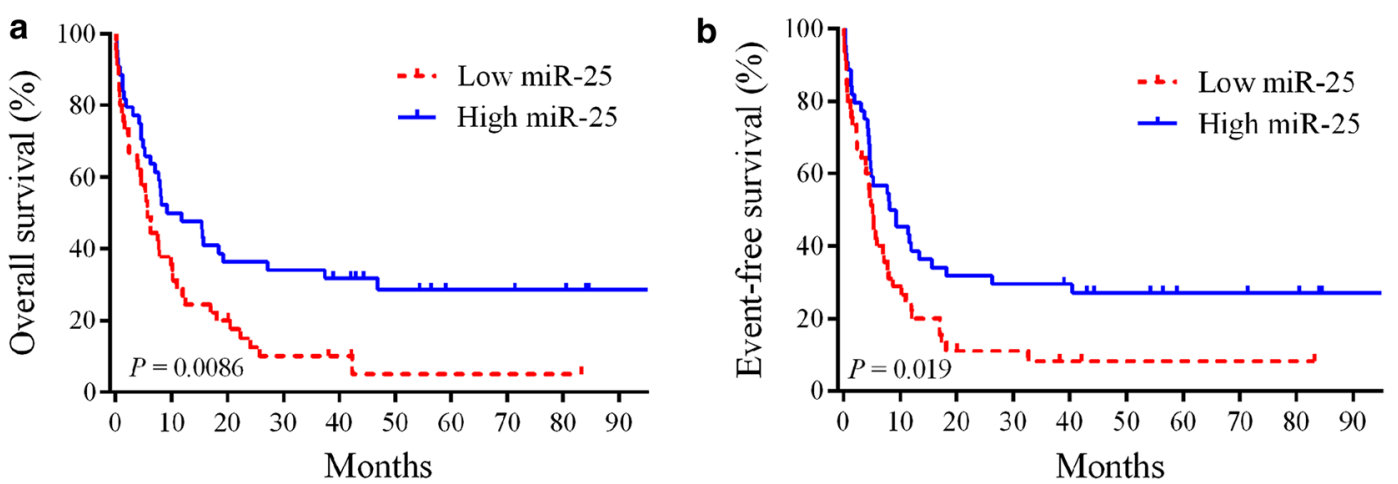

Chemotherapy group

C

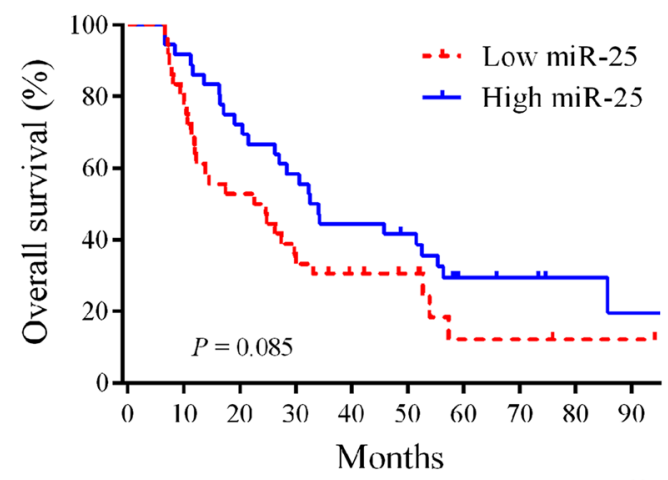

d

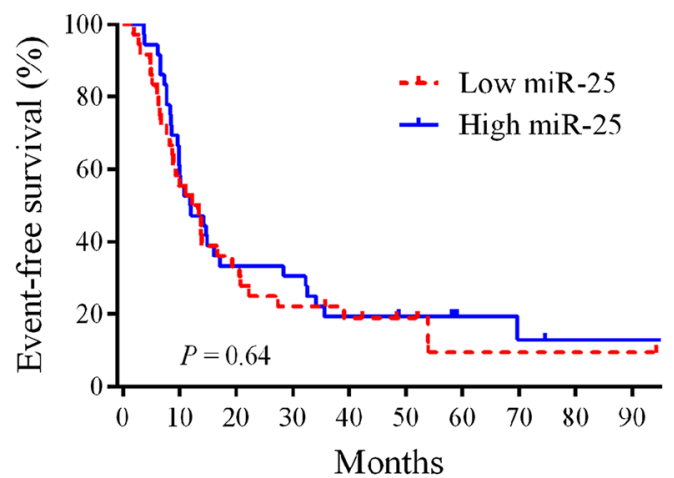

Allo-HCST group

Fig. 1 Kaplan-Meier survival curves of AML patients stratified based on miR-25 expression. $\mathbf{a}, \mathbf{b}$ In the chemotherapy group, the high miR-25 expressers had significantly prolonged OS and EFS $(n=90)$ compared with low miR-25 expressers. $\mathbf{c}$, d There were no significant differences in patients undergoing allo-HSCT between high and low miR-25 groups $(n=72)$

into two groups according to the median miR-25 expression levels. In the downregulated miR-25 group, the AML cases who received allo-HSCT showed significantly longer EFS $(\mathrm{HR}=0.515,95 \% \mathrm{CI} 0.327-0.831, P=0.0069)$ and $\mathrm{OS}(\mathrm{HR}=0.405,95 \%$ CI $0.250-0.639, P=0.0002)$ relative to cases who underwent standard chemotherapy alone (Fig. 2a, b). For the upregulated miR-25 group, no obvious differences in EFS $(P=0.969)$ and OS $(P=0.364)$ were observed among the allo-HSCT and chemotherapy regimens. Thus, the AML patients showing downregulated miR-25 may benefit from treatment with allo-HSCT.

\section{Biological insights into miR-25 profiles in AML}

To generate insights into the molecular mechanism of miR-25, we analyzed a gene expression signature that was connected with miR-25 expression among AML cases. An association between the expression of 205 genes and miR-25 was observed. Among these genes, 145 were negatively correlated and 60 were positively correlated with the expression of miR-25 (Fig. 3).
MiR-25 expression was inversely correlated with the expression of HOXA and HOXB, as well as the HOX cofactor MEIS1. Notably, these genes are crucial for the leukemogenesis and self-renewal capacities of AML [8, $16,17]$. Furthermore, we discovered that the expression of miR-25 was negatively connected with the levels of the PRDM16, Which involved in AML translocation [18]; CD97, an EGF-TM7 receptor [19]; IRAK1, which activates NF- $\mathrm{BB}$ pathways by the interaction with TRAF6 [20]; NFKB2, a pro-inflammatory response gene [21]; MYH9, which predicts unfavorable outcome of AML [22]; HDAC11, a epigenetic regulator. Notably, MYH9 was a in silico predicted target of miR-25.

Gene Ontology showed that genes involved in cellular metabolic process, system development, immune system process, transcription, hematopoietic or lymphoid organ development, hemopoiesis and myeloid cell differentiation were markedly overrepresented among differentially expressed genes associated with miR-25 expression (Table 4). 
Table 2 Univariate and multivariate analyses in patients treated with chemotherapy

\begin{tabular}{|c|c|c|c|c|}
\hline \multirow[t]{2}{*}{ Variables } & \multicolumn{2}{|l|}{ EFS } & \multicolumn{2}{|l|}{ os } \\
\hline & HR $(95 \% \mathrm{Cl})$ & $P$-value & HR $(95 \% \mathrm{Cl})$ & $P$-value \\
\hline \multicolumn{5}{|l|}{ Univariate analyses } \\
\hline MiR-25 (high vs low) & $0.598(0.376-0.951)$ & 0.030 & $0.556(0.347-0.890)$ & 0.015 \\
\hline WBC (<20 vs $\geq 20 \times 109 / L)$ & $0.939(0.594-1.484)$ & 0.786 & $0.936(0.591-1.484)$ & 0.779 \\
\hline FLT3-ITD (positive vs negative) & $1.242(0.693-2.224)$ & 0.467 & $1.192(0.665-2.136)$ & 0.555 \\
\hline NPM1 (mutated vs wild) & $1.168(0.721-1.893)$ & 0.527 & $1.044(0.640-1.704)$ & 0.862 \\
\hline DNMT3A (mutated vs wild) & $1.491(0.909-2.446)$ & 0.114 & $1.432(0.868-2.362)$ & 0.160 \\
\hline RUNX1 (mutated vs wild) & $1.464(0.700-3.064)$ & 0.312 & $1.591(0.759-3.335)$ & 0.219 \\
\hline ITDH1 (mutated vs wild) & $1.043(0.452-2.405)$ & 0.922 & $0.908(0.366-2.254)$ & 0.836 \\
\hline ITDH2 (mutated vs wild) & $0.981(0.487-1.977)$ & 0.956 & $0.991(0.492-1.995)$ & 0.979 \\
\hline \multicolumn{5}{|l|}{ Multivariate analyses } \\
\hline MiR-25 (high vs low) & $0.561(0.333-0.943)$ & 0.029 & $0.502(0.296-0.851)$ & 0.011 \\
\hline WBC (<20 vs $\geq 20 \times 109 / L)$ & $0.884(0.537-1.456)$ & 0.629 & $0.927(0.563-1.527)$ & 0.766 \\
\hline FLT3-ITD (positive vs negative) & $1.489(0.778-2.848)$ & 0.229 & $1.578(0.815-3.054)$ & 0.176 \\
\hline NPM1 (mutated vs wild) & $0.877(0.476-1.615)$ & 0.674 & $0.760(0.411-1.405)$ & 0.382 \\
\hline DNMT3A (mutated vs wild) & $1.421(0.787-2.568)$ & 0.244 & $1.416(0.787-2.550)$ & 0.246 \\
\hline RUNX1 (mutated vs wild) & $1.730(0.768-3.897)$ & 0.186 & $1.805(0.805-4.050)$ & 0.152 \\
\hline ITDH1 (mutated vs wild) & $1.141(0.448-2.904)$ & 0.782 & $1.074(0.397-2.906)$ & 0.889 \\
\hline ITDH2 (mutated vs wild) & $1.039(0.480-2.251)$ & 0.922 & $1.042(0.483-2.248)$ & 0.916 \\
\hline
\end{tabular}

EFS event-free survival, OS overall survival, WBC white blood cell

Table 3 Univariate and multivariate analyses in patients treated with allo-HSCT

\begin{tabular}{|c|c|c|c|c|}
\hline \multirow[t]{2}{*}{ Variables } & \multicolumn{2}{|l|}{ EFS } & \multicolumn{2}{|l|}{ OS } \\
\hline & $\mathrm{HR}(95 \% \mathrm{Cl})$ & $P$-value & $\mathrm{HR}(95 \% \mathrm{Cl})$ & $P$-value \\
\hline \multicolumn{5}{|l|}{ Univariate analyses } \\
\hline MiR-25 (high vs low) & $0.886(0.553-1.473)$ & 0.641 & $0.625(0.364-1.073)$ & 0.088 \\
\hline WBC (<20 vs $\geq 20 \times 109 / L)$ & $1.530(0.910-2.571)$ & 0.108 & $0.949(0.554-1.628)$ & 0.851 \\
\hline FLT3-ITD (positive vs negative) & $1.873(1.020-3.437)$ & 0.043 & $1.998(1.053-3.788)$ & 0.034 \\
\hline NPM1 (mutated vs wild) & $0.913(0.515-1.619)$ & 0.755 & $0.879(0.478-1.617)$ & 0.678 \\
\hline DNMT3A (mutated vs wild) & $1.106(0.615-1.989)$ & 0.737 & $1.269(0.686-2.347)$ & 0.447 \\
\hline RUNX1 (mutated vs wild) & $1.375(0.650-2.907)$ & 0.404 & $2.253(1.046-4.849)$ & 0.038 \\
\hline ITDH1 (mutated vs wild) & $0.985(0.498-1.949)$ & 0.966 & $0.810(0.382-1.718)$ & 0.582 \\
\hline ITDH2 (mutated vs wild) & $0.569(0.227-1.425)$ & 0.229 & $0.931(0.368-2.357)$ & 0.880 \\
\hline \multicolumn{5}{|l|}{ Multivariate analyses } \\
\hline MiR-25 (high vs low) & $0.788(0.421-1.476)$ & 0.457 & $0.510(0.266-0.978)$ & 0.043 \\
\hline WBC (<20 vs $\geq 20 \times 109 / L)$ & $1.343(0.756-2.386)$ & 0.314 & $0.827(0.450-1.519)$ & 0.540 \\
\hline FLT3-ITD (positive vs negative) & $2.222(1.044-4.729)$ & 0.038 & $2.201(0.951-5.096)$ & 0.065 \\
\hline NPM1 (mutated vs wild) & $0.586(0.280-1.227)$ & 0.156 & $0.560(0.249-1.259)$ & 0.161 \\
\hline DNMT3A (mutated vs wild) & 1.058 (0.549-2.037) & 0.867 & 1.514 (0.774-2.963) & 0.226 \\
\hline RUNX1 (mutated vs wild) & $1.483(0.620-3.545)$ & 0.376 & $2.671(1.114-6.402)$ & 0.028 \\
\hline ITDH1 (mutated vs wild) & $1.265(0.535-2.944)$ & 0.592 & $0.781(0.305-1.999)$ & 0.606 \\
\hline ITDH2 (mutated vs wild) & $0.524(0.183-1.498)$ & 0.228 & $0.499(0.175-1.424)$ & 0.194 \\
\hline
\end{tabular}

EFS event-free survival, OS overall survival, WBC white blood cell

\section{Discussion}

AML has been considered to occur as the result of genetic abnormalities, including chromosomal rearrangements, gene deregulations and mutations [23]. The deregulated expression of microRNAs in AML can influence cell proliferation, survival and 

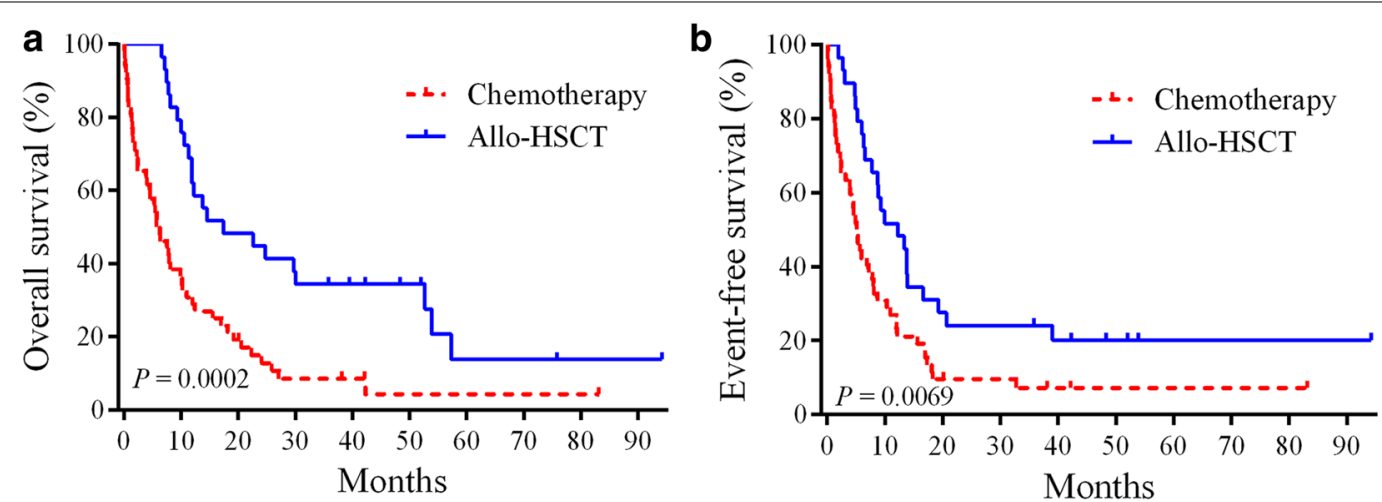

Low miR-25 expression group
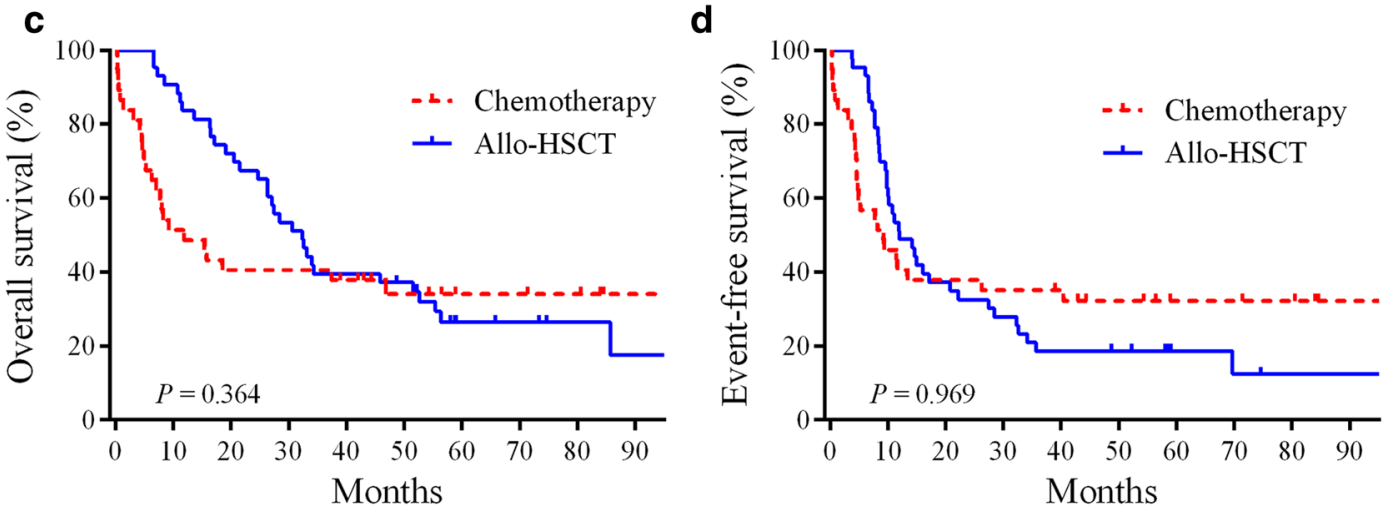

High miR-25 expression group

Fig. 2 Allo-HSCT treatment circumvents the unfavorable outcomes of AML patients showing downregulated miR-25 expression. $\mathbf{a}$, b A total of 162 cases were placed into two groups according to the median miR-25 expression levels. In the downregulated miR-25 group, the Kaplan-Meier survival curves of AML patients classified based on chemotherapy $(n=52)$ and allo-HSCT $(n=29)$ treatment. $\mathbf{c}, \mathbf{d}$ In the upregulated miR-25 group, the Kaplan-Meier survival curves of AML patients classified based on chemotherapy $(n=38)$ and allo-HSCT $(n=43)$ treatment

hematopoietic differentiation [24]. The association of microRNAs with prognosis in heterogeneous patients with AML is still largely unclear. In this evaluation, the upregulated of miR-25 was determined to be an independently favorable prognosticator of AML cases who were administered chemotherapy. Furthermore, alloHSCT may overcome the poor prognosis of AML cases with low miR-25 expression.

A correlation between aberrant miRNA expression and AML prognosis has been established [25, 26]. However, most of previous microRNA markers is restricted to AML without cytogenetic abnormalities. In our study, univariate and multivariate analyses demonstrated that miR-25 is an independently biomarker for cases administered chemotherapy. High miR-25 expression can predict favorable outcome. The prognostic role of miR-25 is different with previously established prognostic factors in a heterogeneous population of AML. MiR-25, as an independent outcome predictor, may improve the current clinical risk-based classification of patients with AML.
To further understand the biological insight into the molecular mechanism underlying miR-25, we identified genes significantly correlated with miR-25 expression. We discovered that the expression of miR-25 negatively connected with the levels of PRDM16, HOXAs, HOXBs, MEIS1, CD97, IRAK1, NFKB2 and MYH9. HOXA and HOXB gene clusters are the common characters of AML $[27,28]$. Of these genes, HOXB4 is positively involved in the renewal of hematopoietic stem cell $[29,30]$. A previous study has shown that HOXA9 contributes to the proliferation, apoptosis, and differentiation processes of leukemia [31]. In addition, HOXA9 has been correlated with poor AML prognosis [32]. Prior evaluations have revealed that IRAK1 may be utilized as a therapeutic target for AML, and TRAF6 may be used to activate pathways such as NFKB, MAPK, and AKT [20, 33]. PRDM16, also known as MEL1, is highly homologous to MDS1/EVI1. High expression of PRDM16 can predict the adverse outcome of AML [18]. Moreover, MYH9 has also been predicted as a direct target of miR-25. High expression of MYH9 can induce 


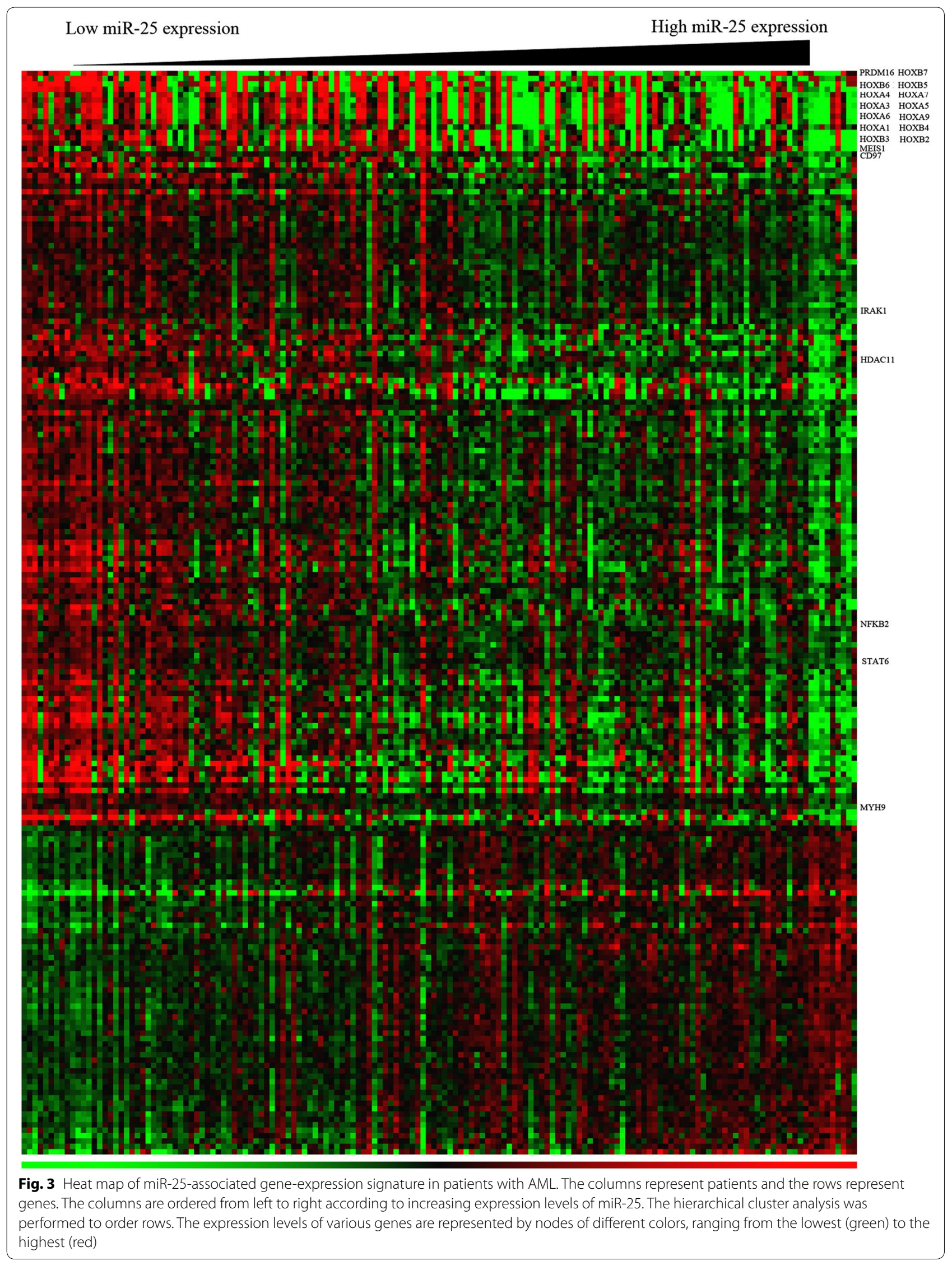


Table 4 Gene ontology terms of biological processes in the miR-25 associated expression profile

\begin{tabular}{|c|c|c|c|}
\hline GO ID & GO terms & $\begin{array}{l}\text { Percentage of members of the } G O \text { term } \\
\text { present in the miR- } 25 \text { profile }\end{array}$ & $P$-value FDR \\
\hline GO:0031323 & Regulation of cellular metabolic process & 46.9 & 0.029 \\
\hline GO:0048522 & Regulation of cellular process & 40.3 & 0.029 \\
\hline GO:0048731 & System development & 37.2 & 0.039 \\
\hline GO:0010604 & Regulation of macromolecule metabolic process & 28.5 & 0.013 \\
\hline GO:0002376 & Immune system process & 26.5 & 0.004 \\
\hline GO:0045893 & Regulation of transcription & 16.8 & 0.037 \\
\hline GO:0048534 & Hematopoietic or lymphoid organ development & 14.7 & $<0.001$ \\
\hline GO:0002520 & Immune system development & 14.7 & $<0.001$ \\
\hline GO:0030097 & Hemopoiesis & 13.7 & $<0.001$ \\
\hline GO:0001501 & Skeletal system development & 9.6 & 0.012 \\
\hline GO:0030099 & Myeloid cell differentiation & 8.1 & 0.009 \\
\hline
\end{tabular}

GO Gene Ontology

resistant to chemotherapy and predict poor clinical outcome in AML [22]. Taken together, the miR-25-associated gene-expression profiling analyses provide insights into the leukemogenic role of genes that are either direct or indirect targets of miR-25. Therefore, the miR-25-associated geneexpression signature analysis give novel insights into the oncogenic role of these genes. These miR-25-related genes could contribute to the chemotherapeutic responses of AML patients.

The FMS-like tyrosine kinase 3 (FLT3) gene is pivotal to hematopoietic stem cell proliferation and differentiation [34]. FLT3 mutations take a great account of most frequent genetic aberrations in AML [35]. FLT3-ITD mutation is one of FLT3 mutations, which can keep the tyrosine kinase persistently active, and result in the abnormal proliferation of leukemic cells. Mutations in the FLT-ITD gene have been associated with higher risk for relapse and poor OS and EFS [36]. Consistent with the conclusion, our data suggested that FLT3-ITD mutation is a poor outcome marker in patients undergoing allo-HSCT. These analysis results indicate that allo-HSCT cannot overcome all adverse prognosis of molecular markers. The findings of this study have revealed that allo-HSCT circumvents the poor chemotherapy outcomes that are related to downregulated miR-25 expression. Thus, low miR-25 expression may be employed as a predictor of adverse prognoses among patients who received chemotherapy, as well as identify patients who require strategies in selecting the best treatment regimen, i.e., chemotherapy and/or allo-HCST.

\section{Conclusion}

In conclusion, high expression of miR-25 was identified to independently predict favorable survival in a highly heterogeneous population of patients with AML. Our findings may offer more information for the therapeutic strategies and the prediction of patients with AML, which may improve the survival and reduce the relapse of them. More importantly, allo-HSCT circumvents poor chemotherapeutic outcomes in cases with downregulated miR-25. The expression levels of miR-25 may thus be utilized in determining whether chemotherapy or allo-HSCT is the optimal treatment regimen for a specific AML patient.

\section{Abbreviations}

AML: acute myeloid leukemia; TCGA: The Cancer Genome Atlas; WHO: World Health Organization; Allo-HSCT: allogeneic hematopoietic stem cell transplantation; OS: overall survival; EFS: event-free survival.

\section{Acknowledgements}

Not applicable.

\section{Authors' contributions}

$M N, Y F$ and NZ designed and performed the computational analyses. TS, $\mathrm{HZ}, \mathrm{RW}, \mathrm{YY}, \mathrm{RY}, \mathrm{QW}$ and JC contributed to statistical analyses. $\mathrm{XL}, \mathrm{YL}$ and $\mathrm{KX}$ designed and wrote the manuscript. All authors read and approved the final manuscript.

\section{Funding}

The research was supported by National Natural Science Foundation of China (81670142, 81772658, 81870163); Jiangsu Provincial Key Research and Development Program (BE2017638, BE2017636); Natural Science Foundation of Jiangsu Province (BK20180104); The Foundation of Jiangsu Province Six Talents Peak (2017-WSN-120); Jiangsu Qing Lan Project for Mingshan Niu; Postgraduate Research \& Practice Innovation Program of Jiangsu Province (SJCX18_0704 SJKY19_2110).

\section{Availability of data and materials}

The datasets supporting the conclusions of this article are included within this article. Clinical data for all patients, including the treatment approach and outcomes data, are publicly accessible from the TCGA website (https://cance rgenome.nih.gov).

\section{Ethics approval and consent to participate}

All data sets were derived from The Cancer Genome Atlas (TCGA) data portal and are publicly accessible from the TCGA website. The patients were enrolled in a single-institution tissue banking protocol approved by the human studies 
committee at Washington University. Written informed consent was obtained from all patients.

\section{Consent for publication}

Not applicable.

\section{Competing interests}

The authors declare that they have no competing interests.

\author{
Author details \\ ${ }^{1}$ Blood Diseases Institute, Affiliated Hospital of Xuzhou Medical University, \\ Xuzhou Medical University, Xuzhou, Jiangsu, China. ${ }^{2}$ Department of Hematol- \\ ogy, Affiliated Hospital of Xuzhou Medical University, Xuzhou, Jiangsu, China. \\ ${ }^{3}$ Institute of Nervous System Diseases, Xuzhou Medical University, Xuzhou, \\ Jiangsu, China. ${ }^{4}$ School of Life Science \& Medicine, Dalian University of Tech- \\ nology, Panjin, China.
}

Received: 17 March 2019 Accepted: 29 April 2019 Published online: 07 May 2019

\section{References}

1. Ding L, Ley TJ, Larson DE, Miller CA, Koboldt DC, Welch JS, et al. Clonal evolution in relapsed acute myeloid leukaemia revealed by wholegenome sequencing. Nature. 2012;481(7382):506-10.

2. Papaemmanuil E, Gerstung M, Bullinger L, Gaidzik VI, Paschka P, Roberts ND, et al. Genomic classification and prognosis in acute myeloid leukemia. N Engl J Med. 2016;374(23):2209-21.

3. Niu M, Shen Y, Qi J, Liu X, Sang W, Wu Q, et al. Effects of realgar $\left(\mathrm{As}_{4} \mathrm{~S}_{4}\right)$ on degradation of PML-RARA harboring acquired arsenic-resistance mutations. Ann Hematol. 2017;96(11):1945-8.

4. Bullinger L, Dohner K, Dohner H. Genomics of acute myeloid leukemia diagnosis and pathways. J Clin Oncol. 2017;35(9):934-46.

5. Chen X, Xie H, Wood BL, Walter RB, Pagel JM, Becker PS, et al. Relation of clinical response and minimal residual disease and their prognostic impact on outcome in acute myeloid leukemia. J Clin Oncol. 2015;33(11):1258-64.

6. Khwaja A, Bjorkholm M, Gale RE, Levine RL, Jordan CT, Ehninger G, et al. Acute myeloid leukaemia. Nat Rev Dis Primers. 2016;2:16010.

7. Nowek K, Sun SM, Dijkstra MK, Bullinger L, Dohner H, Erkeland SJ, et al. Expression of a passenger miR-9* predicts favorable outcome in adults with acute myeloid leukemia less than 60 years of age. Leukemia. 2016;30(2):303-9.

8. Schwind S, Maharry K, Radmacher MD, Mrozek K, Holland KB, Margeson $\mathrm{D}$, et al. Prognostic significance of expression of a single microRNA, miR181a, in cytogenetically normal acute myeloid leukemia: a Cancer and Leukemia Group B study. J Clin Oncol. 2010;28(36):5257-64.

9. Sun SM, Rockova V, Bullinger L, Dijkstra MK, Dohner H, Lowenberg B, et al. The prognostic relevance of miR-212 expression with survival in cytogenetically and molecularly heterogeneous AML. Leukemia. 2013;27(1):100-6.

10. Eisfeld AK, Marcucci G, Maharry K, Schwind S, Radmacher MD, Nicolet D, et al. miR-3151 interplays with its host gene BAALC and independently affects outcome of patients with cytogenetically normal acute myeloid leukemia. Blood. 2012;120(2):249-58.

11. Qu J, Li M, Zhong W, Hu C. Prognostic role of microRNA-25 in cancers: evidence from a meta-analysis. Int J Clin Exp Med. 2015;8(8):12921-7.

12. Wang $M$, Yang $Y O$, Jin $Q$, Shang L, Zhang J. Function of miR-25 in the invasion and metastasis of esophageal squamous carcinoma cells and bioinformatical analysis of the miR-106b-25 cluster. Exp Ther Med. 2018;15(1):440-6.

13. Yan W, Xu L, Sun Z, Lin Y, Zhang W, Chen J, et al. MicroRNA biomarker identification for pediatric acute myeloid leukemia based on a novel bioinformatics model. Oncotarget. 2015;6(28):26424-36.

14. Garzon R, Volinia S, Liu CG, Fernandez-Cymering C, Palumbo T, Pichiorri F, et al. MicroRNA signatures associated with cytogenetics and prognosis in acute myeloid leukemia. Blood. 2008;111(6):3183-9.

15. Zhang $H$, Zhang N, Wang R, Shao T, Feng Y, Yao Y, et al. High expression of miR-363 predicts poor prognosis and guides treatment selection in acute myeloid leukemia. J Transl Med. 2019;17(1):106.
16. Argiropoulos B, Humphries RK. Hox genes in hematopoiesis and leukemogenesis. Oncogene. 2007;26(47):6766-76.

17. Eklund EA. The role of HOX genes in malignant myeloid disease. Curr Opin Hematol. 2007;14(2):85-9.

18. Yamato G, Yamaguchi H, Handa H, Shiba N, Kawamura M, Wakita $\mathrm{S}$, et al. Clinical features and prognostic impact of PRDM16 expression in adult acute myeloid leukemia. Genes Chromosomes Cancer. 2017;56(11):800-9.

19. Wobus M, Bornhauser M, Jacobi A, Krater M, Otto O, Ortlepp C, et al. Association of the EGF-TM7 receptor CD97 expression with FLT3-ITD in acute myeloid leukemia. Oncotarget. 2015;6(36):38804-15.

20. Beverly LJ, Starczynowski DT. IRAK1: oncotarget in MDS and AML. Oncotarget. 2014;5(7):1699-700.

21. Morikawa M, Nakano S, Mitsui N, Murasawa H, Masuki S, Nose H. Effects of dried tofu supplementation during interval walking training on the methylation of the NFKB2 gene in the whole blood of older women. J Physiol Sci. 2017;68:749-57.

22. Yu M, Wang J, Zhu Z, Hu C, Ma Q, Li X, et al. Prognostic impact of MYH9 expression on patients with acute myeloid leukemia. Oncotarget. 2017;8(1):156-63.

23. Grimwade D, Ivey A, Huntly BJ. Molecular landscape of acute myeloid leukemia in younger adults and its clinical relevance. Blood. 2016;127(1):29-41.

24. Dell'Aversana C, Giorgio C, D'Amato L, Lania G, Matarese F, Saeed S, et al. miR-194-5p/BCLAF1 deregulation in AML tumorigenesis. Leukemia. 2018;32(2):573.

25. Shivarov V, Dolnik A, Lang KM, Kronke J, Kuchenbauer F, Paschka P, et al. MicroRNA expression-based outcome prediction in acute myeloid leukemia: novel insights through cross-platform integrative analyses. Haematologica. 2016;101(11):e454-6.

26. Yang C, Shao T, Zhang H, Zhang N, Shi X, Liu X, et al. MiR-425 expression profiling in acute myeloid leukemia might guide the treatment choice between allogeneic transplantation and chemotherapy. J Transl Med. 2018;16(1):267.

27. Spencer DH, Young MA, Lamprecht TL, Helton NM, Fulton R, O'Laughlin $\mathrm{M}$, et al. Epigenomic analysis of the HOX gene loci reveals mechanisms that may control canonical expression patterns in AML and normal hematopoietic cells. Leukemia. 2015;29(6):1279-89.

28. Kontro M, Kumar A, Majumder MM, Eldfors S, Parsons A, Pemovska T, et al. HOX gene expression predicts response to $\mathrm{BCL}-2$ inhibition in acute myeloid leukemia. Leukemia. 2017;31(2):301-9.

29. Antonchuk J, Sauvageau G, Humphries RK. HOXB4-induced expansion of adult hematopoietic stem cells ex vivo. Cell. 2002;109(1):39-45.

30. Miyake N, Brun AC, Magnusson M, Miyake K, Scadden DT, Karlsson S. HOXB4-induced self-renewal of hematopoietic stem cells is significantly enhanced by p21 deficiency. Stem Cells. 2006;24(3):653-61.

31. Collins C, Wang J, Miao H, Bronstein J, Nawer H, Xu T, et al. C/EBPalpha is an essential collaborator in Hoxa9/Meis1-mediated leukemogenesis. Proc Natl Acad Sci USA. 2014;111(27):9899-904.

32. Collins CT, Hess JL. Role of HOXA9 in leukemia: dysregulation, cofactors and essential targets. Oncogene. 2016;35(9):1090-8.

33. Rhyasen GW, Bolanos L, Fang J, Jerez A, Wunderlich M, Rigolino C, et al. Targeting IRAK1 as a therapeutic approach for myelodysplastic syndrome. Cancer Cell. 2013;24(1):90-104.

34. Shlush LI, Zandi S, Mitchell A, Chen WC, Brandwein JM, Gupta V, et al. Identification of pre-leukaemic haematopoietic stem cells in acute leukaemia. Nature. 2014;506(7488):328-33.

35. Stone RM, Mandrekar SJ, Sanford BL, Laumann K, Geyer S, Bloomfield CD, et al. Midostaurin plus chemotherapy for acute myeloid leukemia with a FLT3 mutation. N Engl J Med. 2017;377(5):454-64.

36. Brunet S, Labopin M, Esteve J, Cornelissen J, Socie G, lori AP, et al. Impact of FLT3 internal tandem duplication on the outcome of related and unrelated hematopoietic transplantation for adult acute myeloid leukemia in first remission: a retrospective analysis. J Clin Oncol. 2012;30(7):735-41.

\section{Publisher's Note}

Springer Nature remains neutral with regard to jurisdictional claims in published maps and institutional affiliations. 